\title{
Kidney Injury in COVID-19: an Emerging Concern to the Clinician
}

\author{
Nurshad Ali $^{1}$ (1) $\cdot$ Shakil Mahmood ${ }^{2}$ \\ Accepted: 21 August 2020 / Published online: 24 August 2020 \\ (C) Springer Nature Switzerland AG 2020
}

To the Editor,

COVID-19 outbreak has created a serious health concern worldwide. Respiratory systems are the main target of SARS-CoV-2 infection; however, it can affect multiple organ systems in the human body $[1,2]$. At the beginning of the pandemic, little attention has been paid to the incidence of acute kidney injury (AKI) in COVID-19 patients. However, there is evidence that AKI is prevalent in COVID-19 patients with severe disease courses. Therefore, kidney injury in COVID-19 is an emerging concern to the clinicians.

Some recent studies have shown kidney injury in severe COVID-19 patients [3-5]. A recent study reported that patients with elevated baseline serum creatinine were admitted in the ICU and required more mechanical ventilation than patients with normal serum creatinine levels, indicating that pre-existing kidney dysfunction on admission increases the disease severity [3]. Kidney injury was observed in some severe patients, even those who had no pre-existing kidney disease [3]. The authors also observed that kidney injury on admission and the progression of AKI stage 2 during hospitalization were associated with a greater risk of hospital mortality [3]. Few more studies have also reported a significant association between kidney failure and death in COVID-19 patients $[4,5]$. A recent review reported AKI in up to $25 \%$ of severe COVID-19 patients, especially in those

This article is part of the Topical Collection on COVID-19

Nurshad Ali

nur_rubd@yahoo.com; nali-bmb@sust.edu

1 Department of Biochemistry and Molecular Biology, Shahjalal University of Science and Technology, Sylhet 3114, Bangladesh

2 Department of Biochemistry, Gonoshasthaya Samaj Vittik Medical College, Savar, Dhaka 1344, Bangladesh with pre-existing comorbidities [6]. AKI was associated with higher mortality rates in COVID-19 patients, especially when renal replacement therapy was required [6].

The impact of COVID-19 on the renal system remains unclear and needs to be elucidated. Current knowledge suggests some possible mechanisms that could explain the kidney injury in severe COVID-19 patients. These include direct pathogenic effects of the virus on kidney cells through the angiotensin-converting enzyme 2 (ACE2)-dependent pathway. Recent data showed that ACE2 is more highly expressed in the kidney than in the lungs and heart [6]. Secondly, elevated levels of pro-inflammatory cytokines and pneumoniarelated hypoxia may be associated with renal damage in COVID-19. Thirdly, certain drugs used in the treatment may also have adverse effects on the renal system. Furthermore, COVID-19-related microangiopathy and hemophagocytic macrophage activation may also cause kidney injury in severe disease courses.

Up to now, there is no specific treatment for AKI in COVID-19 [7], although several medications are being used in the treatment of COVID-19, and their effects on AKI are still unknown. Kidney injury worse hypertension and diabetes in severe disease courses and co-existing of these medical conditions can increase hospital mortality. Therefore, severe COVD-19 patients with kidney injury require more attention during hospitalization. Moreover, COVID-19 patients who did not develop an AKI, but who had protein and/or blood in their urine, need to be monitored regularly since they are at increased risk of developing chronic- and end-stage renal disease. Further clinical studies focusing on AKI in severe patients are desperately needed to predict the risk of AKI and elucidate the exact mechanisms of kidney injury in COVID-19.

Authors' Contribution NA conceived the idea and drafted the letter. SM contributed with subsequent versions. All authors approved the final submitted version. 


\section{Compliance with Ethical Standards}

Conflict of interest The authors declare that they have no conflict of interest.

Ethical Approval Ethics approval is not applicable.

\section{References}

1. Tsang KW, Ho PL, Ooi GC, Yee WK, Wang T, Chan-Yeung M, et al. A cluster of cases of severe acute respiratory syndrome in Hong Kong. N Engl J Med. 2003;348:1977-85.

2. Ali N. Is SARS-CoV-2 associated with liver dysfunction in COVID19 patients? Clin Res Hepatol Gastroenterol. 2020: S2210740120301406.

3. Cheng Y, Luo R, Wang K, Zhang M, Wang Z, Dong L, et al. Kidney disease is associated with in-hospital death of patients with COVID19. Kidney Int. 2020;97:829-38.
4. Li Z, Wu M, Yao J, Guo J, Liao X, Song S, et al. Caution on kidney dysfunctions of COVID-19 Patients. preprint. Infect Dis (except HIV/AIDS). 2020. https://doi.org/10.1101/2020.02.08.20021212.

5. Xu S, Fu L, Fei J, Xiang H-X, Xiang Y, Tan Z-X, et al. Acute kidney injury at early stage as a negative prognostic indicator of patients with COVID-19: a hospital-based retrospective analysis. preprint. Urology. 2020. https://doi.org/10.1101/2020.03.24.20042408.

6. Gabarre P, Dumas G, Dupont T, Darmon M, Azoulay E, Zafrani L. Acute kidney injury in critically ill patients with COVID-19. Intensive Care Med. 2020;46:1339-48. https://doi.org/10.1007/ s00134-020-06153-9.

7. Ronco C, Reis T, Husain-Syed F. Management of acute kidney injury in patients with COVID-19. Lancet Respir Med. 2020;8:73842.

Publisher's Note Springer Nature remains neutral with regard to jurisdictional claims in published maps and institutional affiliations. 